\title{
Detrusor Overactivity May Be a Prognostic Factor for Better Response to Combination Therapy Over Monotherapy in Male Patients With Benign Prostatic Enlargement and Storage Lower Urinary Tract Symptoms
}

\author{
Vasileios Sakalis ${ }^{1}$, Vasileios Sfiggas ${ }^{2}$, Ioannis Vouros ${ }^{2}$, George Salpiggidis ${ }^{2}$, Athanasios Papathanasiou ${ }^{2}$, Apostolos Apostolidis ${ }^{3}$ \\ ${ }^{1}$ Department of Urology, Agios Pavlos General Hospital, Thessaloniki, Greece \\ ${ }^{2}$ Department of Urology, Hippokration General Hospital, Thessaloniki, Greece \\ ${ }^{3}$ The 2nd Department of Urology, Papageorgiou General Hospital, Aristotle University of Thessaloniki, Thessaloniki, Greece
}

Purpose: Several trials have shown that in men with overactive bladder (OAB) and benign prostate enlargement (BPE), the combination of an $\alpha$-blocker with an anticholinergic is superior to $\alpha$-blocker monotherapy. The purpose of this study is to explore whether urodynamic detrusor overactivity (DO) affects clinical outcomes in men with BPE and OAB.

Methods: This is a post hoc analysis of a prospective, randomized trial, designed to evaluate the changes of morphometric parameters of the prostate following monotherapy or combination therapy in men with BPE-OAB. The initial study recruited men aged $\geq 50$ years, with BPE and predominantly storage lower urinary tract symptoms (LUTS). Patients were randomized to receive tamsulosin monotherapy or a combination of tamsulosin and solifenacin for 26 weeks. All participants completed a 3-day bladder diary and the International Prostate Symptom Score (IPSS), and then underwent pressure-flow and ultrasonographic studies. Data were stratified and analysed based on the urodynamic observation of DO at baseline (DO vs. non-DO). The primary endpoint was the mean change in urgency episodes from baseline. Secondary outcomes were the changes in nocturia, total IPSS, and urodynamic parameters.

Results: Sixty-nine men were initially randomized, but only 60 men were included in this analysis. Urgency episodes significantly improved in men with $\mathrm{DO}$ who received combination therapy compared to the DO monotherapy subgroup $(\mathrm{P}=0.04)$ or to the non-DO combination treatment subgroup $(\mathrm{P}=0.038)$. Nocturia also improved in the $\mathrm{DO}$ combination treatment subgroup as compared to the non-DO combination subgroup $(\mathrm{P}=0.037)$. The 24-hour frequency and total IPSS significantly improved from baseline without significant differences among the subgroups.

Conclusions: The present study suggests that baseline DO could be a prognostic factor for a better response to combination therapy over monotherapy in men with BPE and storage LUTS.

Keywords: Urinary bladder, Overactive; Benign prostate hyperplasia; Lower urinary tract symptoms; Urgency; Detrusor overactivity

- Research Ethics: The study was approved by the Institutional Review Board and the Ethics Committees of both sites and followed Good Clinical Practice guidelines. The initial trial registration number of this randomized study is ISRCTN 11552705.

- Conflict of Interest: No potential conflict of interest relevant to this article was reported.

Corresponding author: Vasileios Sakalis (iD https://orcid.org/0000-0003-0132-224X Department of Urology, Agios Pavlos General Hospital, Ethnikis Antistasis 161, Kalamaria, 551 32, Thessaloniki, Greece

Email: vsakkalis@hotmail.com

Submitted: May 30, 2020 / Accepted after revision: July 18, 2020
This is an Open Access article distributed under the terms of the Creative Commons Attribution Non-Commercial License (https://creativecommons.org/licenses/by-nc/4.0/) which permits unrestricted non-commercial use, distribution, and reproduction in any medium, provided the original work is properly cited. 


\section{INTRODUCTION}

The links among overactive bladder (OAB), detrusor overactivity (DO), and bladder outlet obstruction (BOO) are not fully understood. There is evidence that in patients with BOO, bladder dysfunction is the result of structural alterations such as detrusor muscle hypertrophy and hyperplasia, increased electrical coupling between cells, partial denervation, super-sensitivity to acetylcholine, increased expression of nerve growth factors and reorganization of C-fiber-mediated spinal micturition reflexes [1]. Peripheral and spinal afferent plasticity related to increased bladder nerve growth factor expression may be a partial explanation for the association between $\mathrm{BOO}$ and $\mathrm{OAB}$ [2].

The prevalence of $\mathrm{OAB}$ in men is estimated at $10 \%-16 \%$; more than half will exhibit DO during pressure-flow studies [3]. It has been proposed that urgency in the absence of DO is not a separate entity, but rather part of the same disease spectrum [4]. The clinical features of bladder dysfunction in patients with DO are fundamentally different from those of patients with urgency and frequency due to increased bladder sensation [5].

Numerous randomized trials have shown that combination treatment of an anticholinergic with an $\alpha$-blocker is superior to a-blocker monotherapy in improving symptoms and quality of life (QoL) in men with $\mathrm{OAB}$ and BOO. Combination treatment was also found to improve urodynamic parameters, such as maximum cystometric capacity and volume until the first involuntary detrusor contraction without major changes in maximum flow rate (Qmax) and postvoid residual (PVR) [6-9]. Alpha-blockers and antimuscarinics are efficient in patients with BOO and OAB, respectively $[10,11]$. Nevertheless, data regarding the efficacy of monotherapy or combination treatment in men with $\mathrm{BOO}$ and $\mathrm{DO}$ are still missing.

Previous studies have analysed data from multicentre, placebo-controlled, randomized trials (TIMES, ADAM) and stratified patients by prostate size and prostate-specific antigen (PSA) levels to assess predictors of the efficacy and safety of tamsulosin monotherapy versus combination therapy with tolterodine versus placebo $[12,13]$. We hypothesized that $\mathrm{DO}$ may be a predictor of responsiveness to therapy and clinical outcomes in men with benign prostatic enlargement (BPE) and OAB. To examine this hypothesis, we conducted a posthoc analysis of our pilot randomized trial, assessing the efficacy of tamsulosin monotherapy versus combination with solifenacin in men with OAB due to BPE [14].

\section{MATERIALS AND METHODS}

\section{Study Design}

This is a post hoc analysis of a prospective, randomized study conducted at 2 centers between October 2013 and June 2015 . The initial study was designed to evaluate changes in the morphometric parameters of the prostate following a-blocker monotherapy or combination of an $\alpha$-blocker with an anticholinergic [14]. The study was approved by the Institutional Review Board and the Ethics Committees of both sites and followed Good Clinical Practice guidelines.

Potential candidates were identified during assessment in the male LUTS clinic. Eligible patients were treatment-naïve men aged $\geq 50$ years with prostate volume $\geq 30 \mathrm{~mL}$ and predominantly storage LUTS as defined by the International Prostate Symptom Score (IPSS) (storage subscore $\geq$ voiding subscore and score $\geq 3$ for the urgency question) and at least 3 urgency episodes per 24 hours as documented in a 3-day bladder diary. The recruited men also had a Qmax $\geq 10 \mathrm{~mL} / \mathrm{sec}, \mathrm{PVR} \leq 100$ $\mathrm{mL}$, and PSA $\leq 4 \mathrm{ng} / \mathrm{mL}$ or a prostate biopsy negative for malignancy in cases of a positive digital rectal examination and/or PSA levels 4-10 ng/mL.

Patients with neurogenic lower urinary tract (LUT) dysfunction or a history of urinary tract malignancy were excluded. Other exclusion criteria were: any contraindication to the use of a-blockers or antimuscarinics; metabolic conditions that may affect LUT function; psychiatric illnesses; chronic kidney, hepatic, or cardiac failure; history of urinary stone disease; history of urethral instrumentation (catheterizations, previous cystoscopies, etc.); urethral stricture; acute urinary tract infection (UTI) or history of recurrent UTIs; the use of medications that are known to affect LUT function, including the periodic use of phosphodiesterase type 5 inhibitors; positive urine culture; and microscopic haematuria, proteinuria, or glycosuria. Patients with pyuria were included only after a negative urine culture.

After providing written informed consent, study participants were randomized (1:1) to receive $0.4 \mathrm{mg}$ of tamsulosin (tamsulosin OCAS $0.4 \mathrm{mg}$; Astellas, Tokyo, Japan) monotherapy or combination therapy of $5 \mathrm{mg}$ of solifenacin plus $0.4 \mathrm{mg}$ of tamsulosin OCAS (Astellas) combination therapy. Titration of the solifenacin dose (to $10 \mathrm{mg}$ ) was possible in combination therapy patients who had inadequate symptom control. Every patient was followed up for 26 weeks. The subjects completed a 3-day bladder diary and filled out the IPSS questionnaire at baseline and at week 26. All patients underwent uroflowmetry, 
a pressure-flow study, and transrectal and transabdominal ultrasonography both at baseline and at the end of the study.

\section{Outcome Measures}

For the purposes of this study, data were stratified based on the urodynamic observation of DO at baseline. DO was defined as an involuntary detrusor contraction during the filling phase, which was either spontaneous or provoked [15]. Patients were classified into those with DO and those without DO (non-DO).

The primary endpoint was the mean change of urgency episodes from baseline to week 26 , as recorded in the 3-day bladder diary. The secondary endpoints were mean changes in nocturia, 24-hour frequency, the total IPSS score, the IPSS storage and voiding subscores, and the QoL score. Basic urodynamic parameters such as Qmax, the bladder outlet obstruction index (BOOI), and PVR were evaluated. Safety was also assessed from adverse event reports.

The paired t-test was used to evaluate intragroup differences and the Mann-Whitney test for intergroup differences. The sample size calculation was based on previously published data, and it was found that in order to detect a difference of $\geq 30 \%$, a power of $80 \%$ would be achieved with a sample of 60 randomized subjects. The level of significance for intergroup differences was set at $\mathrm{P}<0.05$.

\section{RESULTS}

A total of 69 men were randomized to receive either monotherapy or combination therapy; 63 men completed the study (monotherapy $\mathrm{N}=31$, combination therapy $\mathrm{N}=32$ ). Three were excluded from this post hoc analysis due to incomplete data (1 from the monotherapy group and 2 from the combination treatment group). Two of these patients would have been allocated to the non-DO group, while the other to the DO group.

The baseline group characteristics are presented in Table 1. There were no apparent differences in any endpoint between groups (DO vs. non-DO) at baseline with the exception of 24hour frequency (10.4 vs. 9.03) and BOOI (66.4 vs. 49.8). Table 2 presents the mean changes in the study outcomes from baseline to week 26.

Urgency episodes significantly improved in men with DO who received combination treatment as compared to the DO

Table 1. Baseline and demographic data

\begin{tabular}{|c|c|c|c|}
\hline Variable & $\mathrm{DO}(\mathrm{n}=30)$ & Non-DO $(\mathrm{n}=30)$ & P-value \\
\hline Age (yr) & $67.60 \pm 9.65(51-86)$ & $68.20 \pm 8.61(50-80)$ & 0.779 \\
\hline PSA (ng/mL) & $1.37 \pm 0.94(0.14-6.2)$ & $1.99 \pm 1.74(0.37-5)$ & 0.472 \\
\hline Prostate volume (mL) & $48.12 \pm 12.87(30-81)$ & $52.97 \pm 21.27(30-95)$ & 0.324 \\
\hline \multicolumn{4}{|l|}{ Bladder diary } \\
\hline Urgency episodes per $24 \mathrm{hr}^{\mathrm{a}}$ & $3.6 \pm 1.7$ & $3.6 \pm 1.6$ & 0.940 \\
\hline Nocturia episodes per $24 \mathrm{hr}$ & $3.6 \pm 1.64$ & $3.77 \pm 1.6$ & 0.703 \\
\hline Frequency per $24 \mathrm{hr}$ & $10.4 \pm 2.8$ & $9.03 \pm 1.92$ & 0.030 \\
\hline \multicolumn{4}{|l|}{ IPSS } \\
\hline Total score & $20.1 \pm 4.35$ & $20.5 \pm 4.28$ & 0.721 \\
\hline Storage subscore & $11.1 \pm 2.17$ & $10.7 \pm 2.43$ & 0.526 \\
\hline Voiding subscore & $9.2 \pm 3.45$ & $9.8 \pm 3.29$ & 0.496 \\
\hline Quality of life & $3.9 \pm 0.91$ & $4.2 \pm 0.94$ & 0.319 \\
\hline \multicolumn{4}{|l|}{ Urodynamic parameter } \\
\hline Qmax $(\mathrm{mL} / \mathrm{sec})$ & $11.9 \pm 4.97$ & $12.9 \pm 5.61$ & 0.779 \\
\hline $\operatorname{PVR}(\mathrm{mL})$ & $32.1 \pm 21.35$ & $35.7 \pm 29.11$ & 0.574 \\
\hline BOOI & $66.4 \pm 32.9$ & $49.8 \pm 25.02$ & 0.020 \\
\hline
\end{tabular}

Values are presented as mean \pm standard deviation (range).

DO, detrusor overactivity; PSA, prostate-specific antigen; IPSS, International Prostate Symptom Score; Qmax, maximum flow rate; PVR, postvoid residual; BOOI, bladder outlet obstruction index.

${ }^{a}$ Urgency episodes identified as a score $\geq 3$ in for IPSS question 4. 
Table 2. Mean changes from baseline in efficacy variables at week 26

\begin{tabular}{|c|c|c|c|c|c|}
\hline \multirow[b]{2}{*}{ Variable } & \multicolumn{2}{|c|}{ DO } & \multicolumn{2}{|c|}{ Non-DO } & \multirow[b]{2}{*}{ P-value ${ }^{a)}$} \\
\hline & $\begin{array}{l}\text { Tamsulosin } \\
(\mathrm{n}=15)\end{array}$ & $\begin{array}{l}\text { Tamsulosin+solifenacin } \\
\qquad(\mathrm{n}=15)\end{array}$ & $\begin{array}{l}\text { Tamsulosin } \\
(\mathrm{n}=15)\end{array}$ & $\begin{array}{l}\text { Tamsulosin+solifenacin } \\
\qquad(\mathrm{n}=15)\end{array}$ & \\
\hline \multicolumn{6}{|l|}{ Bladder diary } \\
\hline Urgency episodes ${ }^{\mathrm{b})}$ & -1.86 & $-2.99^{c)}$ & -1.97 & -1.50 & 0.038 \\
\hline Nocturia & -1.33 & $-2.25^{\mathrm{c})}$ & -1.76 & -1.60 & 0.03 \\
\hline Frequency per $24 \mathrm{hr}$ & -3.38 & -4.63 & -3.42 & -3.38 & 0.12 \\
\hline \multicolumn{6}{|l|}{ IPSS } \\
\hline Total score & -8.29 & -9.88 & -8.21 & -8.07 & 0.098 \\
\hline Storage subscale score & -4.93 & $-6.5^{c)}$ & -3.86 & $-5.64^{c)}$ & 0.177 \\
\hline Voiding subscale score & -3.14 & -3.28 & $-4.57^{\mathrm{d})}$ & -2.36 & 0.248 \\
\hline Quality of life & -1.78 & -2.2 & -1.71 & -2.36 & 0.569 \\
\hline \multicolumn{6}{|l|}{ Urodynamic parameter } \\
\hline $\mathrm{Qmax}(\mathrm{mL} / \mathrm{sec})$ & $2.68^{\mathrm{e})}$ & -0.87 & 0.5 & -0.4 & 0.498 \\
\hline PVR (mL) & $-2.7^{\mathrm{e})}$ & 24.5 & $-2.4^{\mathrm{e})}$ & 25.2 & 0.941 \\
\hline BOOI & -21.0 & -17.6 & -15.5 & -7.0 & 0.214 \\
\hline
\end{tabular}

DO, detrusor overactivity; IPSS, International Prostate Symptom Score; Qmax, maximum flow rate; PVR, postvoid residual; BOOI, bladder outlet obstruction index.

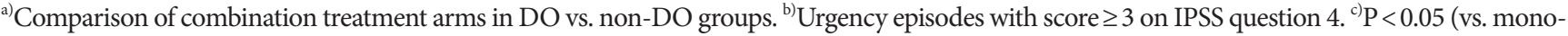
therapy). ${ }^{\text {d) }} \mathrm{P}<0.01$ (vs. combination therapy). ${ }^{\text {e) }} \mathrm{P}<0.05$ (vs. combination therapy).

monotherapy subgroup $(\mathrm{P}=0.04)$, to the non-DO combination treatment subgroup $(\mathrm{P}=0.038)$, and to the non-DO monotherapy subgroup $(\mathrm{P}=0.04)$. The non-DO monotherapy subgroup showed comparable efficacy to the non-DO combination treatment subgroup in terms of improvements in urgency episodes $(\mathrm{P}=0.126)$. In the initial study, both treatment groups showed improvements in urgency episodes as compared to baseline (monotherapy, -3.0 vs. combination, $-3.5 ; \mathrm{P}<0.001$ ), and the intergroup comparison did not reveal any significant differences [14].

Nocturia also improved in men with DO who received combination treatment as compared to the DO monotherapy subgroup $(\mathrm{P}=0.018)$, to the non- $\mathrm{DO}$ combination treatment subgroup $(\mathrm{P}=0.03)$, and to the non-DO monotherapy subgroup $(\mathrm{P}=0.037)$. The non-DO monotherapy subgroup showed comparable efficacy to the non-DO combination treatment subgroup in improving nocturia episodes $(\mathrm{P}=0.599)$. In the analysis of unstratified data, nocturia episodes were reduced in both treatment arms, with near-statistical significance for the combination treatment (monotherapy, -1.5 vs. combination, -2.0 , $\mathrm{P}=0.057)[14]$.

Fig. 1 shows the mean percentage changes in bladder diary variables and IPSS from baseline to week 26 . All variables significantly improved as compared to baseline $(\mathrm{P}<0.05)$ in both treatment groups.

Improvements were found in 24-hour frequency from baseline in all subgroups. Combination therapy was superior to monotherapy in the DO subgroup, without reaching statistical significance $(\mathrm{P}=0.08)$.

The IPSS significantly improved compared to baseline in all subgroups $(\mathrm{P}<0.001)$. Although there were no significant treatment differences in the total IPSS, the DO combination treatment subgroup achieved a greater mean score reduction than the other subgroups (-9.88).

The IPSS storage subscore significantly decreased in both the DO and non-DO combination treatment subgroups as compared to baseline and to the counterpart monotherapy subgroups $(\mathrm{P}=0.045$ and $\mathrm{P}=0.04$, respectively). The IPSS voiding subscore significantly improved in the non-DO monotherapy subgroup as compared to the non-DO combination treatment subgroup $(\mathrm{P}=0.01)$. There were no significant differences in the DO subgroups $(\mathrm{P}=0.457)$.

There were no significant differences in the IPSS QoL question score according to the treatment regimen in the DO or 

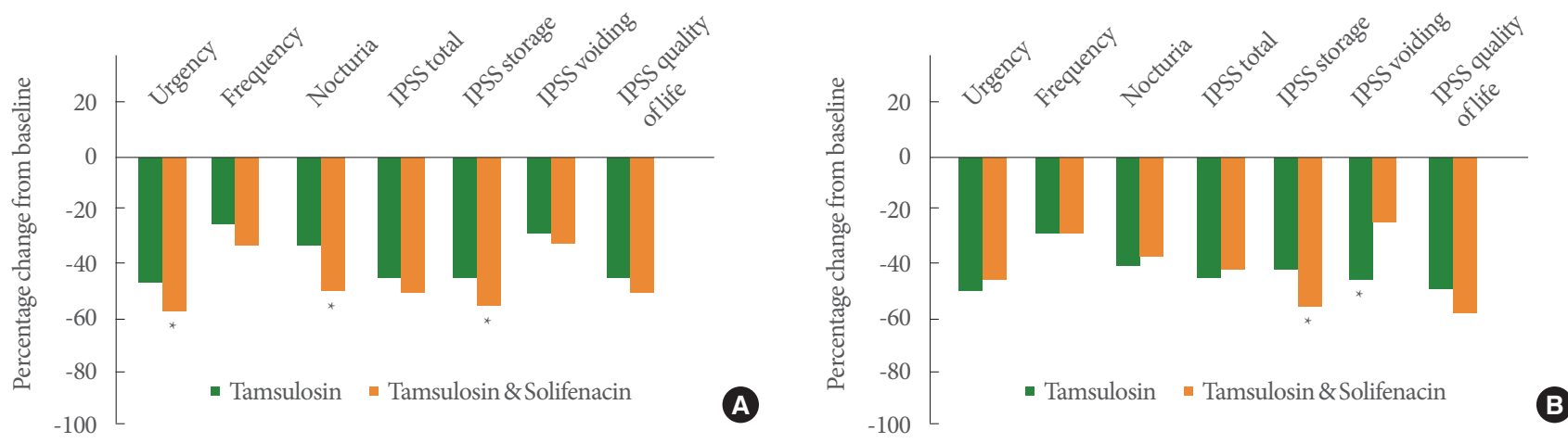

Fig. 1. Percentage change from baseline to week 26 in efficacy variables in patients with baseline $\mathrm{DO}(\mathrm{A})$ and without baseline $\mathrm{DO}$ (B). DO, detrusor overactivity; IPSS, International Prostate Symptom Score. ${ }^{\star} \mathrm{P}<0.05$.

non- $\mathrm{DO}$ groups $(\mathrm{P}=0.281$ and $\mathrm{P}=0.439$, respectively).

PVR significantly improved in monotherapy subjects in both the DO and non-DO groups as compared to combination therapy $(\mathrm{P}=0.023$ and $\mathrm{P}=0.017)$. Qmax also significantly improved in the DO monotherapy subgroup $(\mathrm{P}=0.042)$.

Seven patients were titrated to $10 \mathrm{mg}$ of solifenacin due to poor symptomatic control. Three of them were in the DO subgroup and 4 were in the non-DO subgroup. There were no differences in primary or secondary outcomes from their original subgroups. The post-treatment changes in the titrated DO subgroup were $-2.8,-2.9$, and -7.0 in urgency episodes, nocturia episodes, and the IPSS storage subscale respectively. The corresponding post-treatment changes in the titrated non-DO subgroup were $-1.8,-1.6$, and -6.1 , respectively.

There were no major adverse events with regards to pharmacotherapy. In the tamsulosin group, 4 patients complained of mild light-headedness, 4 patients of anejaculation, and 2 of moderate dyspepsia. In the combination group, 6 patients complained of dry mouth and dry eyes, 5 of constipation, 3 of anejaculation and 2 of dyspepsia. Three men were found to have increased PVR $(>100 \mathrm{~mL})$ at the completion of the study.

\section{DISCUSSION}

The present study showed that baseline DO was a prognostic factor for better response to combination therapy over monotherapy in men with BPE and storage LUTS. Compared to tamsulosin monotherapy, patients receiving combination therapy with tamsulosin and solifenacin reported significant improvements in urgency episodes, nocturia episodes, and the storage IPSS subscore. By contrast, patients with baseline DO receiving tamsulosin monotherapy had significant improve- ments in Qmax and PVR as compared to combination treatment. Both treatment subgroups had similar rates of persistent DO following treatment (46.6\% vs. $40.0 \%)$. It should be noted that both groups showed significant improvements from baseline independent of the presence of baseline $\mathrm{DO}(\mathrm{P}<0.05)$.

The beneficial effects of antimuscarinics for storage LUTS have been well documented in multiple randomized placebocontrolled trials and in several meta-analyses. Antimuscarinics inhibit the afferent (direct or indirect) stimulatory action of acetylcholine, derived from neuronal or nonneuronal sources during bladder filling [16]. At low doses, antimuscarinics could also inhibit afferent activity or noise generated within the detrusor muscle, suppressing idiopathic DO [17]. A small-scale randomized trial in a neurogenic population showed that trospium relieved DO as well [18]. Antimuscarinics are competitive antagonists of muscarinic receptors, but their effect on detrusor contraction is minimal during the voiding phase, due to the massive acetylcholine surge that competes for receptor site binding.

The findings of this secondary analysis are consistent with previous reports, which found that combination therapy of alpha-blockers with an anticholinergic better controlled storage LUTS than monotherapy. Multicenter randomized trials in men with $\mathrm{OAB}$ and BPE have shown that the combination of solifenacin and tamsulosin is superior to tamsulosin monotherapy in IPSS domains, bladder diary parameters, and healthrelated QoL domains $[10,19]$. Unfortunately, the effect of antimuscarinic monotherapy in men with LUTS and BPE is difficult to assess due to the theoretical risk of urinary retention. Recent evidence has suggested that there is a $0.3 \%-0.6 \%$ risk of retention in mixed LUTS populations [20]. It is obvious that small increases in PVR volume $(+25 \mathrm{~mL})$ are unlikely to in- 
crease the risk of retention [20].

Large-scale observational studies in female patients with $\mathrm{OAB}$ did not show any difference in antimuscarinic efficacy according to the presence of DO $[21,22]$. In a trial of a predominantly female $\mathrm{OAB}$ population, where patients were randomized to receive tolterodine or placebo, the investigators concluded that urodynamic assessment is not a prerequisite for antimuscarinic treatment and patients with apparently normal urodynamics responded equally well to treatment as did those with DO [21]. In men with LUTS, such data are still missing.

Another important outcome of this secondary analysis is that in non-DO men, the efficacy of tamsulosin monotherapy was comparable to that of combination therapy in bladder diary parameters, the total IPSS, and the IPSS QoL score. An explanation could be that bladder $\alpha_{1 d}$-adrenergic receptors are associated with storage symptoms and $\alpha_{1 \mathrm{~d}}$ receptors in the spinal cord play a potential role in afferent activity control [23]. Monotherapy was more favourable for improvements in the IPSS voiding subscore and PVR, while combination treatment showed better improvements in the IPSS storage subscore. Changes in bladder diary parameters were significant from baseline $(\mathrm{P}<0.05)$, but the intergroup comparison showed insignificant changes after 26 weeks of treatment in 24-hour frequency (-3.42 vs. -3.38), urgency episodes (-1.97 vs. -1.50$)$, and in nocturia (-1.76 vs. $-1.60)$. The lack of superiority of solifenacin in the non-DO group requires careful interpretation, since the reduction of peripheral resistance induced by tamsulosin may relieve DO in few patients. De Nunzio et al. [24] studied the effect of watchful waiting, medical therapy, or surgery on DO evolution and found minimal improvement after alfuzosin treatment (1 patient improved out of 7).

In animal models of bladder overactivity, tamsulosin exerted an inhibitory effect on neuronal activation in brain and spinal centres associated with micturition control [25]. In addition, tamsulosin appears to have an inhibitory effect on C-fibers, thereby improving storage function [26]. It has been demonstrated that BOO alters bladder $\alpha$-AR subtype composition, while $\beta$-AR responsiveness appears absent or of limited magnitude [27-29]. Hampel et al. [30] reported a change from $\alpha 1 \mathrm{~A}$ to a1D predominance (decrease from $70 \%$ to $23 \%$ for $\alpha 1 \mathrm{~A}$; increase from $25 \%$ to $75 \%$ for a1D) in BOO, suggesting that targeting a1D may provide a new therapeutic approach for storage symptoms associated with BOO.

Nocturia, a frequent symptom in $\mathrm{OAB}$ patients, has a significant impact on QoL [31,32]. Evidence suggests that waking up once per night is associated with minimal bother, whereas waking up to void $\geq 2$ times has a negative impact on the patient's ability to function, causes chronic fatigue and negatively affects QoL [33]. Whilst nocturnal polyuria is the most common cause, bladder storage problems could cause troublesome nocturia $[34,35]$. Hence, it is expected that pharmacotherapy for $\mathrm{OAB}$ would improve not only daytime symptoms, but also nocturia as well, thereby improving QoL.

Our findings suggest that combination therapy is superior to monotherapy for improving nocturia episodes in men with baseline DO. This group showed significantly reduced nocturia frequency as compared to tamsulosin monotherapy $(\mathrm{P}<0.05)$ and to combination treatment group in non-DO men $(\mathrm{P}=0.03)$. The results of the original study did not reveal a firm statistically significant difference between the 2 main therapy groups (-1.5 vs. -2.0$)$. An explanation is that in patients with baseline $\mathrm{DO}$, nocturia could be due to bladder storage problems; therefore, antimuscarinics are more efficient. The existing literature can be confusing on this subject. Although solifenacin monotherapy has been found to significantly reduce nocturia against placebo or against active comparators in mixed $\mathrm{OAB}$ patient populations, the NEPTUNE study, which randomized more than 1,000 male LUTS patients to receive monotherapy with tamsulosin, a combination of tamsulosin and solifenacin (6 mg and $9 \mathrm{mg}$ ), or placebo, could not demonstrate superiority of combination therapy over monotherapy in nocturia, despite improvements in both storage and voiding symptoms as well as QoL measures [10]. In fact, the change in nocturia episodes per 24 hours was insignificant $(\mathrm{P}>0.05)$ in all treatment arms [10].

A strength of our study is the homogeneous population sample. Our patients shared similar symptoms and a reasonable flow. The cutoff for maximum flow was $10 \mathrm{~mL} / \mathrm{sec}$ while the cutoffs for symptoms were $\geq 3$ urgency episodes/24 hours and frequency of $\geq 8$ episodes. The inclusion criteria in most previously published studies were a Qmax of 4-12 mL/sec and $\geq 2$ urgency episodes per 24 hours. However, the lack of a placebo arm and the small sample size may limit the interpretation of the results. Although the original study was adequately powered for the primary outcome, it was not adequately powered to assess subgroup differences. Thus, the results of this secondary analysis should be interpreted with caution. The need for invasive urodynamics is another drawback for the application of our results in clinical practice, and noninvasive clinical correlates for DO would ideally be welcome. In both cases, largescale and adequately powered randomized controlled trials 
might offer greater insights into these issues.

In conclusion, the results of the post hoc analysis of this randomized pilot study suggest that the presence of baseline DO in male patients with storage LUTS and BPE could be a prognostic factor for a better response of symptoms of urgency and nocturia to combination therapy over monotherapy. Therefore, clinicians could directly treat these patients with combination of an $\alpha$-blocker and an anticholinergic, rather than $\alpha$-blocker monotherapy, to achieve earlier symptomatic control.

\section{AUTHOR ORCID}

Vasileios Sakalis

0000-0003-0132-224X

Apostolos Apostolidis

0000-0003-4694-0578

\section{AUTHOR CONTRIBUTION STATEMENT}

- Conceptualization: V Sakalis, $A A$

- Data curation: V Sakalis, V Sfiggas, IV, AA

- Formal analysis: $V$ Sakalis

- Methodology: V Sakalis, $A A$

- Project administration: $A A$

- Visualization: $V$ Sakalis

-Writing-original draft: $V$ Sakalis

- Writing-review \& editing: V Sakalis, GS, AP, AA

\section{REFERENCES}

1. Elbadawi A, Meyer S. Morphometry of the obstructed detrusor. I. Review of the issues. Neurourol Urodyn 1989;8:163-71.

2. Steers WD, Creedon DJ, Tuttle JB. Immunity to nerve growth factor prevents afferent plasticity following urinary bladder hypertrophy. J Urol 1996;155:379-85.

3. Irwin DE, Milsom I, Hunskaar S, Reilly K, Kopp Z, Herschorn S, et al. Population based survey of urinary incontinence, overactive bladde, and other lower urinary tract symptoms in five countries: results of the EPIC study. Eur Urol 2006;50:1306-15.

4. Haylen BT, Chetty N, Logan V, Schulz S, Verity L, Lae M, et al. Is sensory urgency part of the same spectrum of bladder dysfunction as detrusor overactivity? Int Urogynecol J Pelvic floor Dysfunct 2007;18:123-8.

5. Apostolidis A, Gonzales GE, Fowler CJ. Effect of intravesical Resiniferatoxin (RTX) on lower urinary tract symptoms, urodynamic parameters and quality of life of patients with urodynamic increased bladder sensation. Eur Urol 2006;50:1299-305.
6. Blake-James BT, Rashidian A, Ikeda Y, Emberton M. The role of anticholinergics in men with lower urinary tract symptoms suggestive of benign prostatic hyperplasia: a systematic review and metaanalysis. BJU Int 2007;99:85-96.

7. Athanasopoulos A, Gyftopoulos K, Giannitsas K, Fisfis J, Perimenis P, Barbalias G. Combination treatment with an alpha-blocker plus an anticholinergic for bladder outlet obstruction: a prospective, randomized, controlled study. J Urol 2003;169:2253-6.

8. Kaplan SA, Walmsley K, Te AE. Tolterodine extended release attenuates lower urinary tract symptoms in men with benign prostatic hyperplasia. J Urol 2005;174:2273-5, discussion 5-6.

9. Lee JY, Kim HW, Lee SJ, Koh JS, Suh HJ, Chancellor MB. Comparison of doxazosin with or without tolterodine in men with symptomatic bladder outlet obstruction and an overactive bladder. BJU Int 2004;94:817-20.

10. van Kerrebroeck P, Chapple C, Drogendijk T, Klaver M, Sokol R, Speakman M, et al. Combination therapy with solifenacin and tamsulosin oral controlled absorption system in asingle tablet for lower urinary tract symptoms in men: efficacy and safety results from the randomized controlled NEPTUNE trial. Eur Urol 2013; 64:1003-12.

11. Kaplan S, Roehrborn C, Dmochowski R, Rovner E, Wang J, Guan Z. Tolterodine extended release improves overactive bladder symptoms in men with overactive bladder and nocturia. Urology 2006; 68:328-32.

12. Roehrborn CG, Kaplan SA, Jones JS, Wang JT, Bavendam T, Guan Z. Tolterodine extended release with or without tamsulosin in men with lower urinary tract symptoms including overactive bladder symptoms: effects of prostate size. Eur Urol 2009;55:472-81.

13. Chapple CR, Herschorn S, Abrams P, Wang JT, Brodsky M, Guan Z. Efficacy and safety of tolterodine extended release in men with overactive bladder symptoms treated with an a-blocker $a$-blocker: effect of baseline prostate-specific antigen concentration. BJU Int 2010;106:1332-8.

14. Sakalis V, Sfiggas V, Vouros I, Salpiggidis G, Papathanasiou A, Apostolidis A. Combination of solifenacin with tamsulosin reduces prostate volume and vascularity as opposed to tamsulosin monotherapy in patients with benign prostate enlargement and overactive bladder symptoms: results from a randomized pilot study. Int J Urol 2018:25:737-45.

15. Abrams P, Cardozo L, Fall M, Griffiths D, Rosier P. The standardization of terminology in lower urinary tract function: report from the standardization subcommittee of the International Continence Society. Urology 2003;61:37-49.

16. Andersson KE. Antimuscarinic mechanisms and overactive detru- 
sor: an update. Eur Urol 2011;59:377-86.

17. Finney SM, Andersson KE, Gillerspie JI, Stewart LH. Antimuscarinic drugs in detrusor overactivity and the overactive bladder syndrome: motor or sensory actions? BJU Int 2006;98:503-7.

18. Stohrer M, Bauer P, Giannetti BM, Richter R, Burgdorfer H, Murtz G. Effect of trospium chloride on urodynamic parameters in patients with detrusor hyperreflexia due to spinal cord injuries. A multicentre placebo-controlled double-blind trial. Urol int 1991; 47:138-43.

19. Van Kerrebroeck P, Haab F, Angulo JC, Vik V, Katona F, GarciaHernandez A, et al. Efficacy and safety of solifenacin plus tamsulosin OCAS in men with voiding and storage lower urinary tract symptoms: Results from a phase 2, dose-finding study (Saturn). Eur Urol 2013;64:398-407.

20. Abrams P, Kelleher C, Staskin D, Rechberger T, Kay R, Martina R, et al. Combination treatment with mirabegron and solifenacin in patients with overactive bladder: efficacy and safety results from a randomized, double-blind, dose-rabging, phase 2 study (Symphony). Eur Urol 2015:67:577-88.

21. Mallone-Lee JG, Henshaw DJ, Cumminhs K. Urodynamic verification of an overactive bladder is not a prerequisite for antimuscarinc treatment response. BJU Int 2003;92:415-7.

22. Digesu GA, Khular V, Cardozo L, Salvatore S. Overactive bladder symptoms: do we need urodynamics? Neurourol Urodyn 2003;22: 105-8.

23. Michelotti GA, Price DT, Schwinn DA. al-Adrenergic receptor regulation: basic science and clinical implications. Pharmacol Therapeut 2000;88:281-309.

24. De Nunzio C, Franco G, Rocchegiani A, Iori F, Leonardo C, Laurenti $\mathrm{C}$. The evolution of detrusor overactivity after watchful waiting, medical therapy and surgery in patients with bladder outlet obstruction. J Urol 2003;169:535-9.

25. Kim SE, Shin MS, Kim CJ, Park JH, Chung KJ, Jung H, et al. Effects of tamsulosin on urinary bladder function and neuronal activity in the voiding centers of rats with cyclophosphamide-induced overactive bladder. Int Neurourol J 2012;16:13-22.
26. Yokoyama O, Yusup A, Oyama N, Aoki Y, Miwa Y, Akino H. Improvements in bladder storage function by tamsulosin depends on suppression of C-fiber urethral afferent activity in rates. J Urol 2007; 177:771-5.

27. Barendrecht MM, Frazier EP, Vrydag W, Alewijnse AE, Peters SLM, Michel MC. The effect of bladder outlet obstruction on a1an $\beta$-Adrenoceptor expression and function. Neurourol Urodyn 2009;28:349-55.

28. Michel MC. $\beta$-Adrenergic receptor subtypes in the urinary tract. In: Andersson KE, Michel M, editors. Urinary tract. Berlin; Springer; 2011. p. 307-18.

29. Monica FZ, Bricola AA, Bau FR, Freitas LL, Teixeira SA, Muscara $\mathrm{MN}$, et al. Long-term nitric oxide deficiency causes muscarinic supersensitivity and reduces beta(3)-adrenoceptor-mediated relaxation, causing rat detrusor overactivity. Br J Pharmacol 2008;153; 1659-68.

30. Hampel C, Dolber PC, Smith MP, Savic SL, Thuroff JW, Thor KB, et al. Modulation of bladder alphal-adrenergic receptor subtype expression by bladder outlet obstruction. J Urol 2002;167:1513-21.

31. Homma Y, Yamaguchi O, Hayashi K; Neurogenic Bladder Society Committee. Epidemiologic survey of lower urinary tract symptoms in Japan. Urology 2006;68:560-4.

32. Espuna-Pons M, Blasco P, Perez M, Rebollo P. Nocturia in patients with overactive bladder. Arch Esp Urol 2010;63:363-72.

33. Oelke M, Roehrborn C, D’Ancona C, Wilson T, Castro R, Manyak M. Nocturia improvement in the combination of avodart and tamsulosin. World J Urol 2014;32:1133-40.

34. Sakalis VI, Karavitakis M, Bedretdinova D, Bach T, Ruud JLH, Gacci M, et al. Medical treatment of nocturia in men with lower urinary tract symptoms: systematic review by the European Association of Urology Guidelines Panel for male lower urinary tract symptoms. Eur Urol 2017;72:757-69.

35. Clemens JQ, Wiseman JB, Smith AR, Amundsen CL, Yang CC, Bradley MS, et al. Prevalence, subtypes and correlates of nocturia in the symptoms of lower Urinary Tract Dysfunction Research Network cohort. Neurourol Urodyn 2020;39:1098-107. 